\title{
Determination of geometrical and kinematical properties of frontside halo coronal mass ejections (CMEs)
}

\author{
XuePu Zhao \\ W. W. Hansen Experimental Physics Laboratory, Stanford University, Stanford, CA \\ 94305-4085, USA \\ email: xpzhao@solar.stanford.edu
}

\begin{abstract}
Recent studies show that the cone model with a circular cross section can be used to determine the geometrical and kinematical properties only for a class of halo CMEs with the semi-minor axis of the elliptic halo threading the solar disk center. This work shows how to use an improved cone model with an elliptic cross section to determine the geometrical and kinematical properties for another class of halo CMEs with the semi-major axis threading the solar disk center.
\end{abstract}

Keywords. coronal mass ejections (CMEs)

\section{Introduction}

Determination of the geometrical and kinematical properties of halo CMEs is necessary for understanding the cause of halo CMEs. It is also necessary for predicting the geoeffectiveness of frontside halo CMEs. For broadside CMEs, i.e., the CMEs with the latitudinal span of their bright feature in the plane of the sky being less than $120^{\circ}$, the latitudinal span and its bisector are the measure of the angular width and central position angle of the broadside CMEs, respectively. Such geometrical properties for halo CMEs, i.e., the CMEs with the latitudinal span greater than $120^{\circ}$, can not be measured directly from white-light images. The central position of halo CMEs is often assumed to be located near the associated surface activities such as solar flares, though it has been reported that CME-associated flares or active regionsare often located near one leg of CMEs.

The speed profiles of CMEs are often derived by choosing a specific feature in a timelapse movie and tracking its position outward with time. For broadside CMEs the measured velocity and acceleration are nearly radial directed; they are, however, projected against the plane of the sky for halo CMEs.

We have developed a cone model with a circular cross section (Zhao, et al. (2002)) for determining the geometrical and kinematical properties of halo CMEs from coronal images. The present work shows how to use an improved cone model with an elliptic cross section to determine the geometrical and kinematical properties for another class of halo CMEs that can not be modeled using the original cone model.

\section{Halo CMEs with minor axis threading solar disk center}

Halo CMEs are interpreted as the result of the Thompson scattering of Sun's white light by a broad shell or bubble of dense plasma of CMEs along the line-of-sight (Howard, et al. (1982)). Specifically, the broad shell of dense plasma may be a conical shell with a circular cross section, as shown by Figure 4 of Howard, et al. (1982). It has been shown 
recently that the projection of the circular cross section against the plane of the sky has elliptic shape with the semi-minor axis threading the solar disk center (Xie, et al. (2004) and Zhao (2004)). Figure 1 shows the images for the 6 January 1997 halo CME observed at various time, $t$, and the ellipse calculated at corresponding heliocentric distance $r(t)$. The determined angular width and central position of the 6 January 1997 halo CME are shown at the top of the first panel.

\section{Halo CMEs with major axis threading solar disk center}

There are halo CMEs with their semi-major, instead of semi-minor, axis threading the solar disk center, suggesting that the corresponding cross section must be elliptic if the halo CMEs are formed by Thompson scattering of a conical shell of dense plasma. Figure 2 shows the images for the 7 April 1997 halo CME observed at various time, $t$, and the ellipse calculated at corresponding $r(t)$. The determined angular width and central position of the 7 April 1997 halo CME are shown at the top of the first panel.

Figure 3 shows the hight-time scatter-point plot based on the fitting result in Figure 2 and the velocity and acceleration inferred for the 7 April 1997 halo CMEs.

\section{Summary and Disscussion}

Halo CMEs exhibit various shapes. Some show ellipse (including circle), and others show ellipse-like but with ragged structure. Many elliptic halo CMEs may be interpreted as the Thompson scattering of Sun's white light by a conical shell of dense plasma along the line-of-sight (Howard, et al. (1982)). The halo CMEs with ragged structure is interpreted as the deflection of preexisting coronal features by super-Alfvenic CMEs (St. Cyr \& Hundhausen (1998)). The ragged structure may be used to distinguish the deflectionformed halo CME from the scattering-formed halo CME (Sheeley et al. (2000)).

Recent studies have shown that the cone model with a circular cross section can be used to determine geometrical and kinematical properties only for a specific class of scattering-formed elliptic halo CMEs of which the minor axis threading the solar disk center. We show that the scattering-formed elliptic halo CMEs with their major axis threading the solar disk center may be modeled using the cone model with an elliptic cross section, and their geometrical and kinematical properties may also be determined.

There are evidence that the plasma structure for some halo CMEs may be approximated using the spherical shell model (Howard, et al. (1982)) or the ice-cream cone model (Fisher \& Munro (1984)). It is interesting to develop a model for determining the geometrical and kinematical properties for those halo CMEs.

\section{Acknowledgements}

\section{References}

Fisher, R.R. \& Munro, R.H. 1984, Astrophys. J. 280, 428

Howard, R.A., Michels, D.J., Sheeley Jr., N.R., \& Koomen, M.J. 1982, Astrophys. J. 263, L101

Sheeley, Jr., N.R., Hakala, W.N. \& Wang, Y.-M. 2000, J. Geophys. Res. 105, A3, 5081

St. Cyr, O.C. \& Hundhausen, A.J. 1998, in: V.P. Pizzo, T. Holzer, \& D.G. Sime (ed.), Proceedings of the 6th International Solar Wind Conference (Boulder), p. 235

Xie, H., Ofman, L. \& Lawrence, G. 2004, J. Geophys. Res 109, A8

Zhao, X.P., Plunkett, S.P., \& Liu, W. 2002, J. Geophys. Res. 107, A8

Zhao, X.P. 2004, in: A.V. Stepanov, E.E. Benevolenskaya \& A.G. Kosovichev (eds.), MultiWavelength Investigations of Solar Activity, Proceedings of IAU 223, in press 


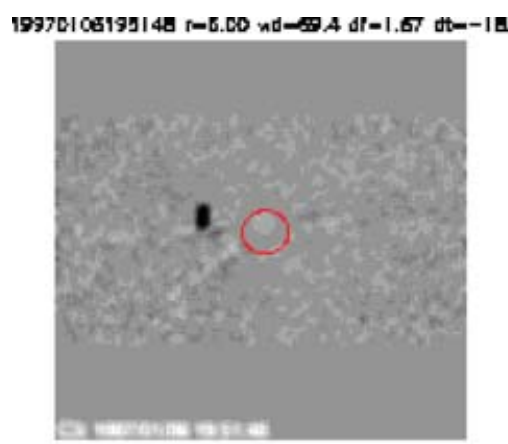

$19970102211000 r=7.00$

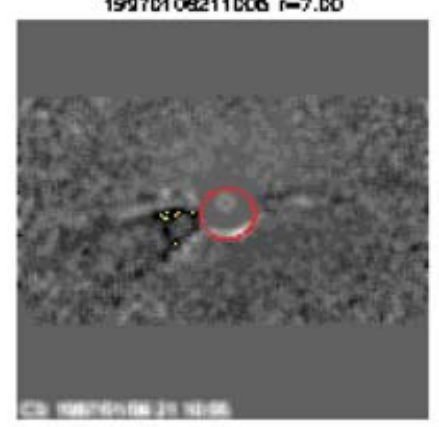

$19270100294800 r=9.00$

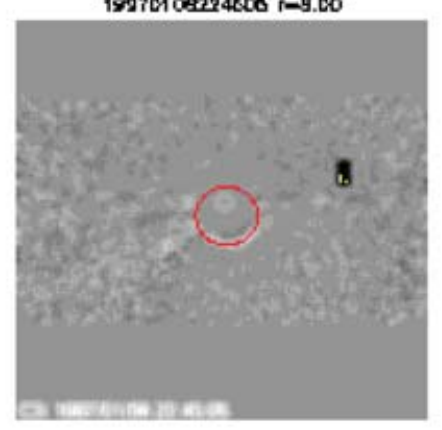

1997010a254506 -0.30

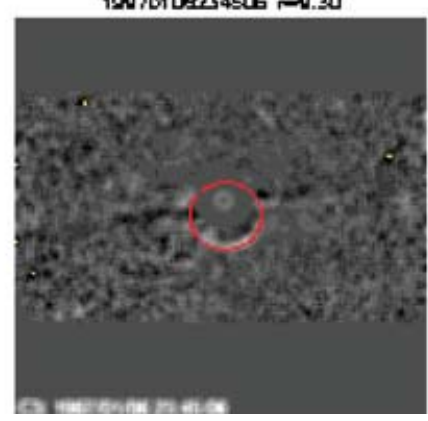

1997010702006 $\mathrm{t}=1000$

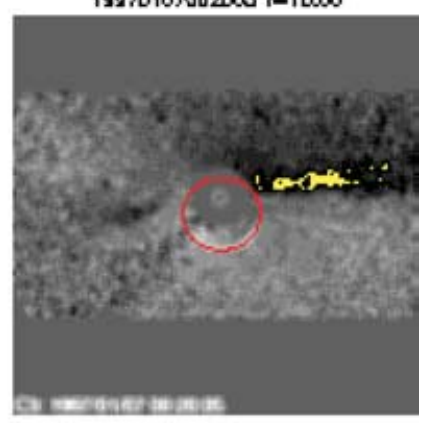

$12970107013606 t=1200$

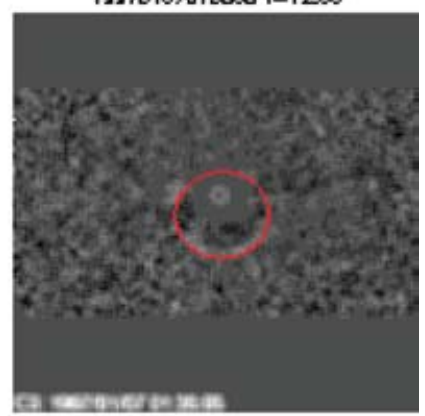

19g70107024006 $r=13.00$

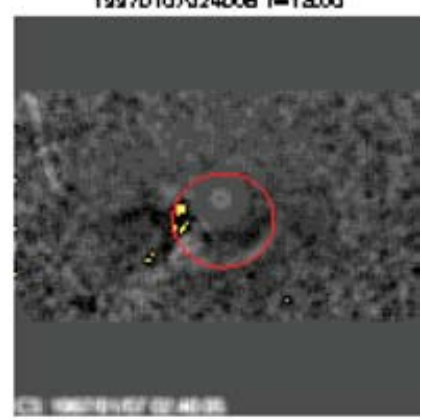

$100 \pi 0107034505$ r 1450

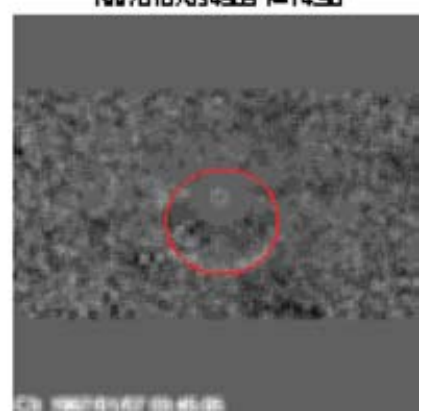

Figure 1. Comparison of the plane-of-sky projection of circular cross sections calculated using the cone model at various heliocentric distances $r(t)$ with the halos observed in difference images at various times, $t$. 

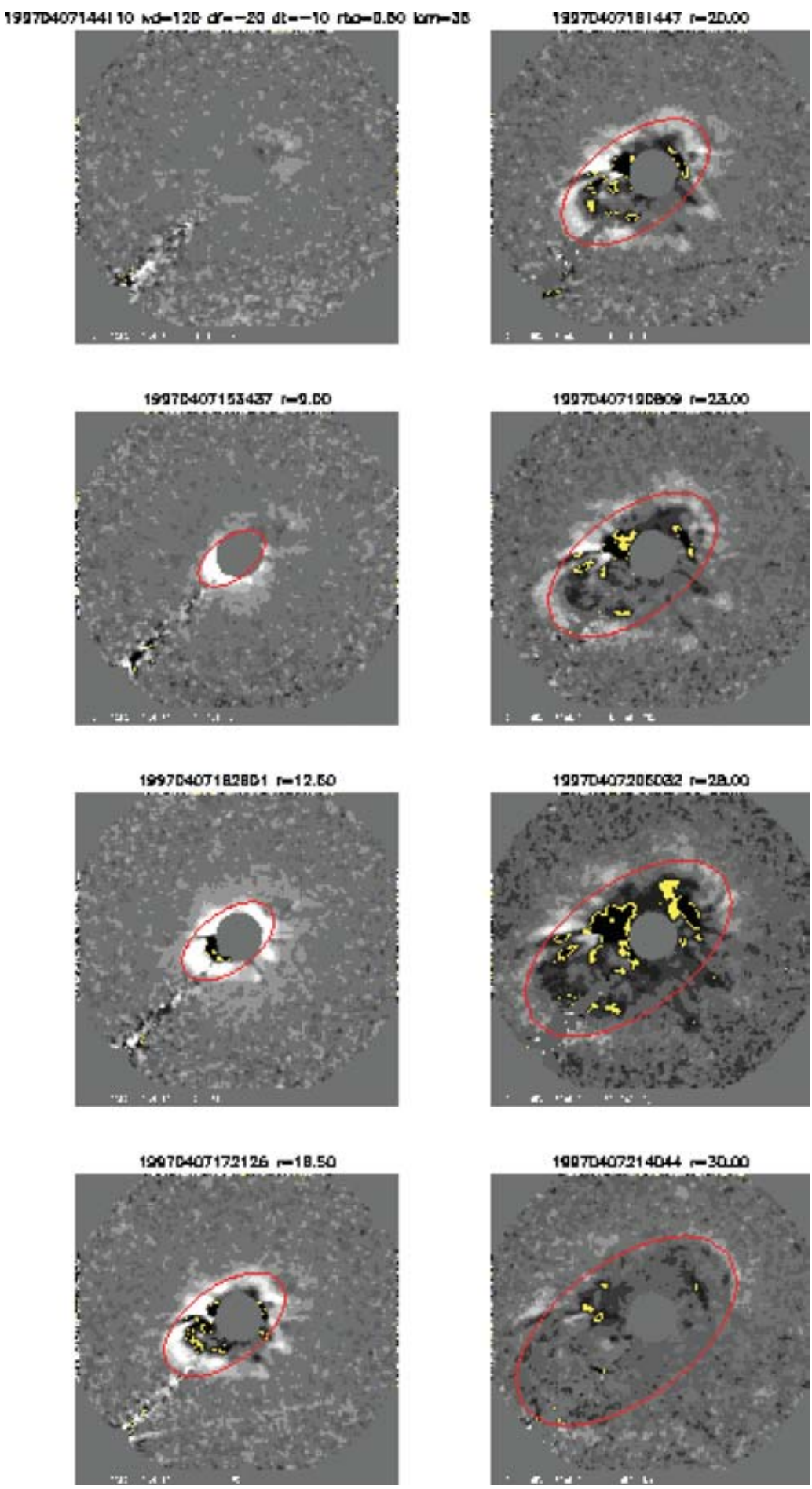

Figure 2. Comparison of the plane-of-sky projection of elliptic cross sections calculated using the cone model at various heliocentric distances $r(t)$ with the halos observed in difference images at various times, $t$. 

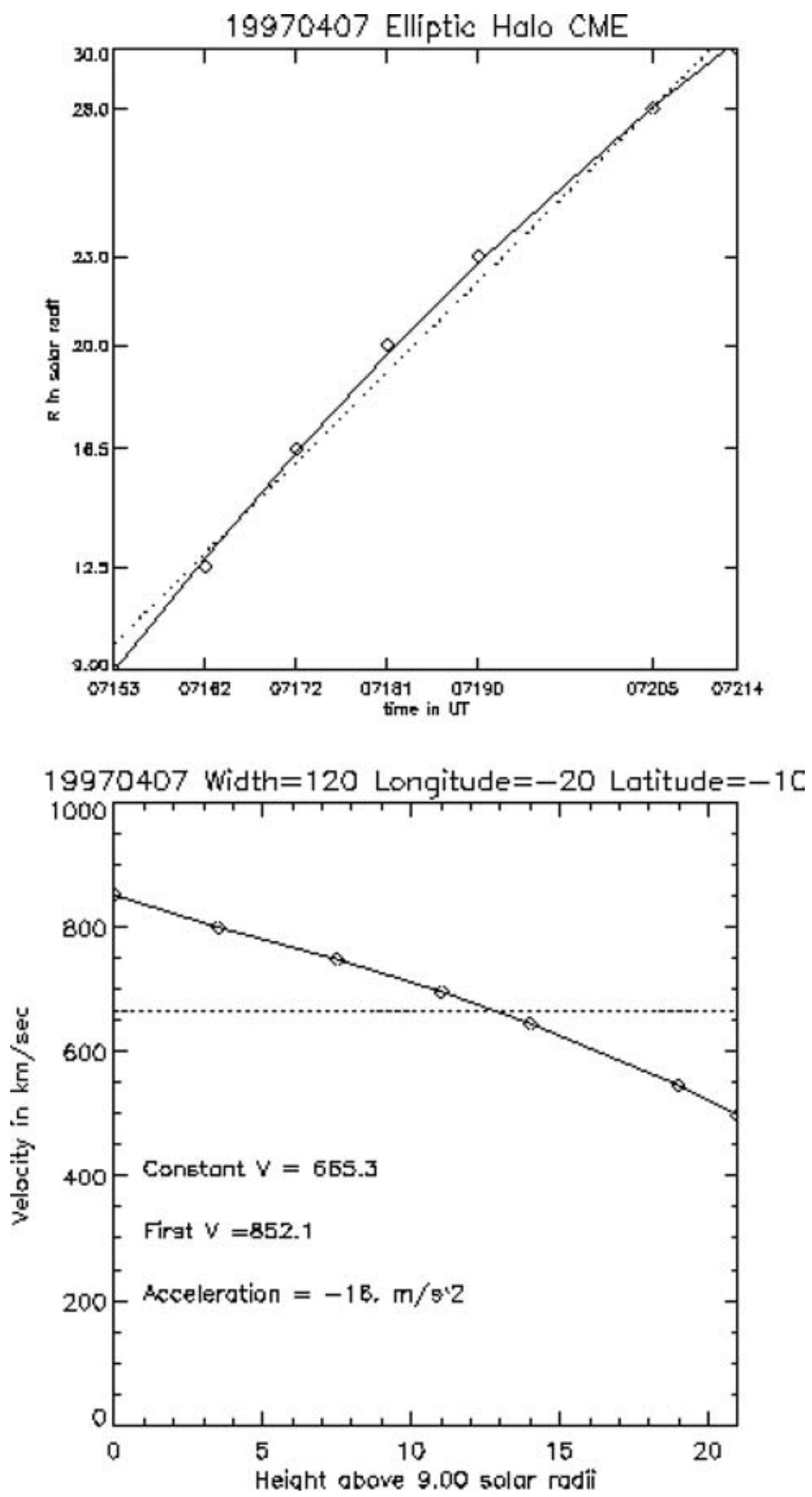

Figure 3. The hight-time scatter-point plot and fitting curve for the 7 April 1997 halo CMEs (the top panel) and the inferred variation of the velocity with hight (the bottom panel). 


\section{Discussion}

P.F. Chen: Have you statistically investigated the CME properties after the correction by your model ?

Zhaо: No, I just examined a few events. The number is not big enough to do any statistical analysis. 